\section{ACDA}

\section{Ärzteverband Deutscher}

Allergologen (ÅDA)

\section{Verantwortlich für den Inhalt}

Prof. Dr. Ludger Klimek

Zentrum für Rhinologie und Allergologie

An den Quellen 10, 65183 Wiesbaden

Tel.: (06 11) 8804799

Fax: (06 11) 308608255

E-Mail: ludger.klimek@allergiezentrum.org

\section{ÄDA-Geschäftsstelle}

Service Systems

Blumenstraße 14, 63303 Dreieich

Tel.: (o 61 03) 62273

Fax: (o 61 03) 697019

E-Mail:info@aeda.de

www.aeda.de

\title{
Kennen Patienten eigentlich die Hyposensibilisierung?
}

m 27. März dieses Jahres wurde im Rahmen der "Sonntagsvorlesungen“" im Berliner Maxim-Gorki-Theater über „Die Pollenallergie“ berichtet. Alle $\mathrm{Zu}-$ hörer erhielten am Eingang das Angebot, nach dem Vortrag einen kurzen Fragebogen auszufüllen. Dies taten 72 Personen mit einem Altersdurchschnitt von 57 Jahren.

Das Ergebnis: 42 (58\%) der Zuhörer gaben an, einen Heuschnupfen zu haben; keine Überraschung beim gewählten Thema. Aber nur 15 (36\%) von ihnen wurde jemals eine Hyposensibilisierung mit der Spritze (subkutane Immuntherapie, SCIT) und noch seltener (acht der von Heuschnupfen Betroffenen, 19\%) mit Tropfen oder Tabletten (sublinguale Immuntherapie, SLIT) vom Arzt angeboten. Auf die Wiederholungsfrage „Hat Ihnen ein Arzt schon einmal eine Hyposensibilisierung angeboten?"“ antworteten nur 16 (38\%) der Betroffenen mit Ja, aber 25 (60\%) mit Nein, eine Person machte keine Angabe.

Fazit: Bei dieser völlig unwissenschaftlichen einfachen Beobachtung zeigt sich erneut, dass selbst interessierte Heuschnupfenpatienten - wer sonst geht am Sonntagvormittag zu einem Vortrag? nur wenig über die Möglichkeit einer Hyposensibilisierung informiert sind. Es gibt viel zu tun: weniger bei den Ärzten als in der Aufklärung der Betroffenen.

Karl-Christian Bergmann, Berlin

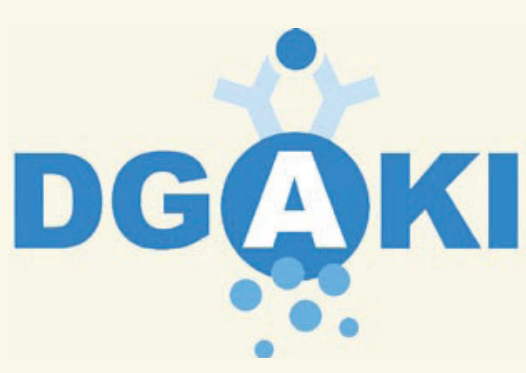

\section{Deutsche Gesellschaft für \\ Allergologie und klinische Immunologie (DGAKI)}

Verantwortlich für die Inhalt

Prof. Dr. Thilo Jakob

Allergieabteilung und Forschergruppe

Allergologie, Universitäts-Hautklinik

Freiburg

Hauptstraße 7,79104 Freiburg

Tel.: (07 61) 2706714

Fax: (07 61) 2706655

E-Mail:thilo.jakob@uniklinik-freiburg.de

\section{DGAKI-Geschäftsstelle}

Gertrud Hammel

Schloss 2, 86482 Aystetten

Tel.: (08 21) 48687864

Fax: (08 21) 48687863

E-Mail: info@dgaki.de

www.dgaki.de

\section{$\square$ Ja $\square$ Nein $\square$ Keine Angabe}

Haben Sie eine Allergie?

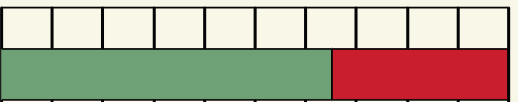

Haben Sie einen Heuschnupfen?

Hat Ihnen ein Arzt schon einmal die Hyposensibilisierung mit Spritzen (SCIT) angeboten?

Hat Ihnen ein Arzt schon einmal die Hyposensibilisierung mit Tropfen oder Tabletten (SLIT) angeboten?

Hat er Sie dabei über den unterschiedlichen Wissenstand bezüglich Wirksamkeit und Verträglichkeit der verschiedenen Behandlungsformen informiert?

Hat Ihnen ein Arzt schon einmal die Hyposensibilisierung angeboten?

Sind Sie gesetzlich versichert?
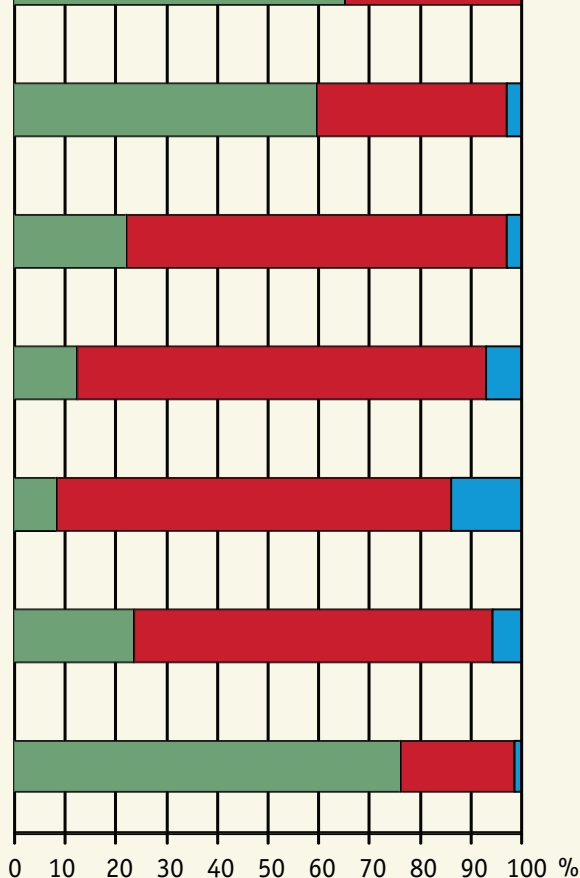

Die Befragung der Zuhörer einer Berliner Sonntagsvorlesung zur Pollenallergie führte zu unerwarteten Ergebnissen: Eine Hyposensibilisierung war nur $\mathbf{3 8 \%}$ der Heuschnupfenpatienten unter ihnen vom Arzt angeboten worden. 